\title{
Increased Basal Ganglia Iron In Striatonigral Degeneration: In Vivo Estimation With Magnetic Resonance
}

\author{
W.R. Wayne Martin, Ted E. Roberts, Frank Q. Ye and Peter S. Allen
}

\begin{abstract}
Background: As many as 20\% of individuals with the clinical diagnosis of Parkinson's disease (PD) do not have the characteristic neuropathologic features of PD at post mortem. The striatonigral degeneration (SND) subtype of multiple system atrophy is one of the categories of pathology which may be incorrectly diagnosed as PD on the basis of clinical presentation. SND may be associated with increased iron deposition in the putamen which can be detected with magnetic resonance imaging. Methods: We have estimated regional brain iron content in a patient with probable SND, using a novel imaging method developed in our laboratory, and have compared the results in this patient to those which we have previously reported in patients with PD and in age-matched controls. Results: We observed that putamenal iron content was increased in our SND patient, beyond the $95 \%$ confidence limit for inclusion in the PD group, even when considering clinical severity. In contrast, pallidal and thalamic iron were within the PD range. Conclusions: The demonstration of increased putamenal iron content may be a useful adjunctive investigative procedure in patients with suspected SND.
\end{abstract}

\begin{abstract}
RÉSUMÉ: Évaluation du contenu en fer des noyaux lenticulaire, caudé, amygdalien et de l'avant-mur au moyen de la résonance magnétique dans la dégénérescence striatonigrale. Introduction: Au moins $20 \%$ des patients qui ont un diagnostic clinique de maladie de Parkinson (MP) n'ont pas les caractéristiques neuropathologiques de la MP à l'autopsie. La dégénérescence striatonigrale (DSN) est un sous-type d'atrophie multisystémique qui est une des pathologies incorrectement diagnostiqées comme MP à cause de sa présentation clinique. La DSN peut être associée à une augmentation du fer dans le putamen qui peut être détectée par la résonance magnétique (RM). Méthodes: Nous avons évalué le contenu régional en fer du cerveau chez un patient probablement atteint de DSN au moyen d'une méthode utilisant la RM développée dans notre laboratoire. Nous avons comparé les résultats chez ce patient à des résultats que nous avons déjà publiés chez des patients atteints de MP et chez des contrôles appariés pour l'âge. Résultats: Nous avons observé que le contenu en fer du putamen était augmenté chez notre patient atteint de DSN, au delà de la limite de confiance de $95 \%$ pour l'inclusion du patient dans le groupe MP, même après considération de la sévérité clinque de la maladie. Pas contre, le contenu en fer du pallidum et du thalamus étaient dans l'écart de ceux de la MP. Conclusion: La démonstration que le contenu en fer est augmenté dans le putamen chez les patients chez qui on soupçonne une DSN peut être utile dans l'investigation de ces patients.
\end{abstract}

Can. J. Neurol. Sci. 1998; 25: 44-47

The diagnosis of Parkinson's disease (PD), although straightforward in many patients, is not without its challenges. As many as $20 \%$ of individuals with this clinical diagnosis do not have the characteristic neuropathologic features of PD at post mortem. ${ }^{1}$ One of the categories of pathology which may be incorrectly diagnosed as PD on the basis of clinical presentation is the family of disorders termed "multiple system atrophy" (MSA). These disorders include striatonigral degeneration (SND), Shy-Drager syndrome, and sporadic forms of olivopontocerebellar atrophy. Magnetic resonance (MR) imaging may help differentiate these disorders from idiopathic PD by virtue of the decreased signal from the basal ganglia which may be evident on $\mathrm{T}_{2}$-weighted images in some patients with MSA. ${ }^{2.3}$ Although numerous factors have a potential effect on $T_{2}$ values, the changes on these images have been generally ascribed to increased iron deposition in the basal ganglia. ${ }^{4}$
More specific MR methods to measure relative regional brain iron levels in living patients with PD have recently been reported. ${ }^{5,6}$ These techniques are relatively insensitive to other factors which shorten $T_{2}$ values and have suggested significant increases in iron content in the substantia nigra ${ }^{5}$ as well as both pallidum and putamen $^{6}$ in idiopathic PD. These increases correlate with the severity of clinical symptomatology. We now report the in vivo estimation of basal ganglia iron content with MR in a patient with a clinical diagnosis of the SND variant of MSA and contrast these findings to those which we have previously reported in PD.

From the Division of Neurology (W.R.W.M., T.E.R.) and the Department of Biomedical Engineering (F.Q.Y., P.S.A.), University of Alberta, Edmonton.

RECEIVED APRIL 16, 1997. ACCEPTED IN FINAL FORM AUGUST 14, 1997.

Reprint requests to: W.R.W. Martin, Movement Disorder Clinic, Glenrose Rehabilitation Hospital, 10230 - 111 Avenue, Edmonton, Alberta, Canada T5G 0B7 


\section{Patient Description}

This right-handed female patient first developed neurological symptoms at age 50, when she observed micrographia. She was diagnosed as having PD shortly afterward and was started on levodopa/carbidopa about one year later because of general bradykinesia. She complained of a continued gradual progression of symptoms, observing that her movements had become generally slow, that her gait had become unsteady and shuffling, and that she had some impairment of dexterity in her upper limbs. By 3 years after symptom onset, she was receiving sustained release levodopa/carbidopa $200 / 50$ qid. She observed a definite improvement of motor symptoms in response to this medication, but reported end-of-dose deterioration with each dose lasting for only about 4 hours.

Neurological examination at this time, 3 years after symptom onset and about 2 hours after receiving a single tablet of sustained release levodopa/carbidopa 200/50, showed normal higher cortical functions with a score of $29 / 30$ on the mini-mental status scale. ${ }^{7}$ There was considerable hypomimia but cranial nerves were otherwise normal. A moderate degree of axial and limb rigidity was present, and there was marked bradykinesia. There was no tremor or other abnormal involuntary movements. Deep tendon reflexes were symmetric, and plantar responses flexor. Finger-nose and heel-shin tests were normal. Her posture was flexed, her gait slow and shuffling, and her postural reflexes impaired. BP was 150/90 when supine and 160/90 when standing.

Over the following 6 months, she experienced rapid deterioration in her speech and swallowing. Bromocriptine was introduced and slowly increased to $5 \mathrm{mg}$ tid without symptomatic benefit. She complained of a lack of mental clarity at this dose and discontinued the bromocriptine. Pergolide was subsequently introduced with some symptomatic benefit at a dosage of $0.75 \mathrm{mg}$ bid.

Symptoms and signs continued to progress over the following 2 years. By 5 years after the initial symptoms, she was virtually mute, requiring a keyboard for communication. She reported that it took 2 hours to eat each meal. There was no evidence of cognitive impairment. Eye movements were normal. There was marked antecollis and considerable drooling. There was severe bradykinesia affecting all voluntary movements and rigidity of all limbs. There was considerable anteropulsion and impairment of postural reflexes to the extent that she required the use of a walker to maintain an upright posture. She had violaceous hands and urinary incontinence. BP was 130/80 when supine and 90/60 when standing. At this time, she was receiving a total of $1000 \mathrm{mg}$ of levodopa and $1.5 \mathrm{mg}$ of pergolide daily with no apparent therapeutic benefit.

\section{Magnetic Resonance Methodology and Results}

About 4 years after the initial development of symptoms, the patient underwent MR imaging using a novel method which we have described in detail previously. This method provides an estimate of regional brain iron concentration, based on the observation that cellular compartmentalization of large paramagnetic susceptibility differences can promote large increases in relaxation rates that are in turn highly sensitive to interecho times of certain MR pulse sequences. ${ }^{8,9}$ We have shown that the

Table: Regional Iron Content, Measured as $\Delta \mathrm{R}_{2}{ }^{\prime}$, in Normal Controls and Parkinson's Disease (mean $\pm S D$ ), and Probable Striatonigral Degeneration.

\begin{tabular}{lccc}
\hline & $\begin{array}{l}\text { Normal } \\
\text { controls } \\
(\mathbf{n}=\mathbf{1 3})\end{array}$ & $\begin{array}{c}\text { Parkinson's } \\
\text { disease } \\
(\mathbf{n}=\mathbf{1 2})\end{array}$ & $\begin{array}{c}\text { Striatonigral } \\
\text { degeneration } \\
(\mathbf{n}=\mathbf{1})\end{array}$ \\
Putamen & $1.29 \pm 0.59$ & $1.83 \pm 0.46^{*}$ & 3.79 \\
Pallidum & $2.13 \pm 0.48$ & $2.83 \pm 0.65^{*}$ & 4.33 \\
Thalamus & $0.26 \pm 0.22$ & $0.49 \pm 0.38$ & 0.39
\end{tabular}

"different from normal controls at $p<0.05$; control and Parkinson's disease values are from reference 6 . echo time dependence of the transverse decay rate $R_{2}^{\prime}\left(1 / T_{2}^{\prime}\right)$ can be used to provide an index of the tissue concentration of iron which is sequestered within cells. ${ }^{10}$ We have also demonstrated, in excised brain tissue, a close linear relationship between this echo time dependence and the direct measurement of iron concentration with atomic absorption spectroscopy. ${ }^{\prime \prime}$ Our methodology yields a single parameter, which we have termed $\Delta \mathrm{R}_{2}^{\prime}$, for each region of interest (ROI). This parameter captures the degree of regional relaxation enhancement, thus providing a quantitative index of tissue iron content.

We compared $\Delta R_{2}{ }^{\prime}$ measurements from pallidum, putamen, and thalamus in this patient with those which we have described previously in 12 patients with idiopathic PD and 13 agematched normal controls. ${ }^{6}$ Quantitative $\Delta R_{2}{ }^{\prime}$ values are listed in the Table. Our patient had a substantial increase in putamenal iron, as indicated by increased $\Delta R_{2}{ }^{\prime}$, in comparison to patients with PD. Because both pallidal and putamenal iron increases
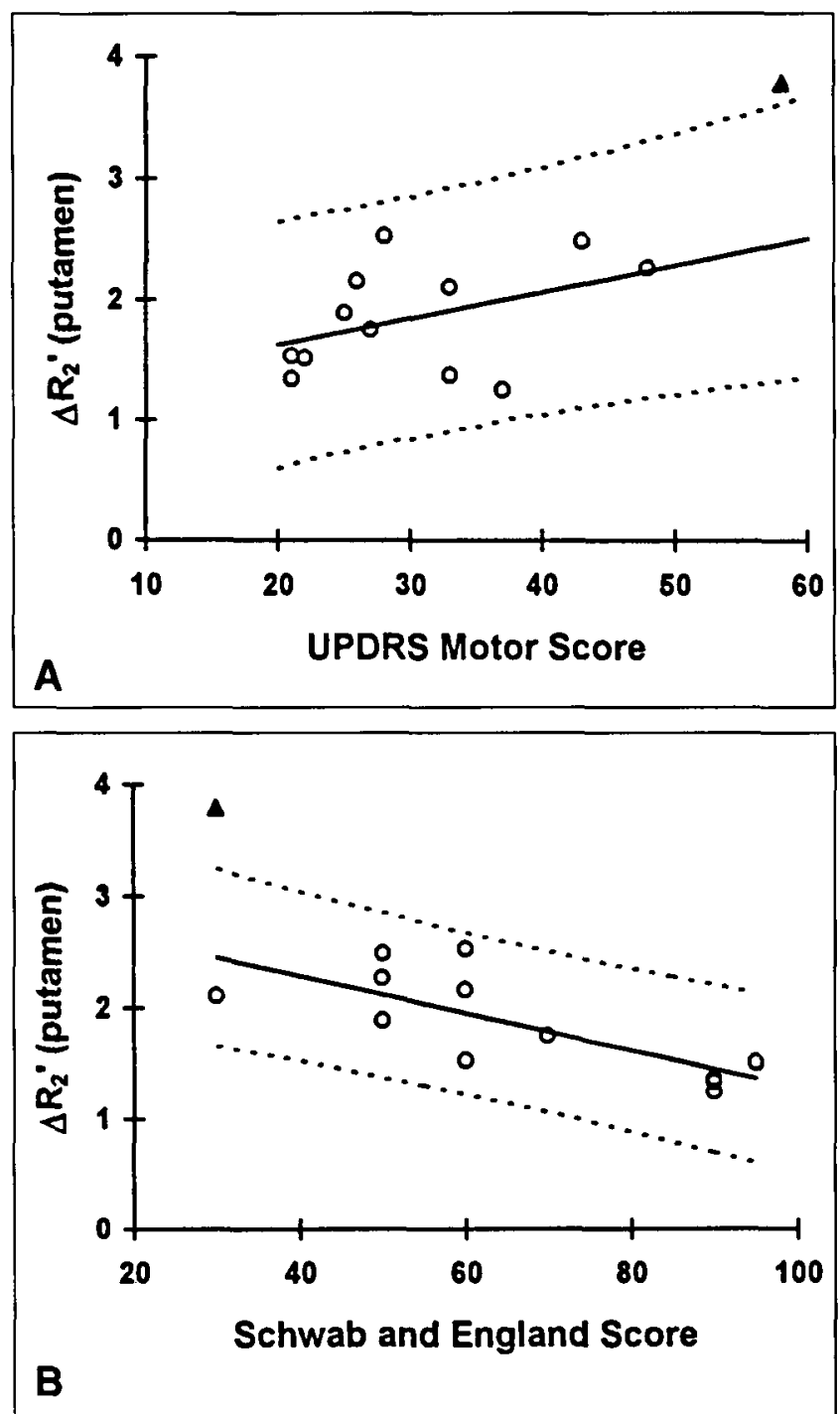

Figure 1: $\Delta R_{2}^{\prime}$ (iron content) in putamen in Parkinson's disease (open circles) and a patient with probable striatonigral degeneration (closed triangle) as a function of UPDRS motor score A) and Schwab and England activities of daily living score B). The regression line and 95\% confidence limits for inclusion in the Parkinson's disease group are indicated. Parkinson's patients are from reference 6. 

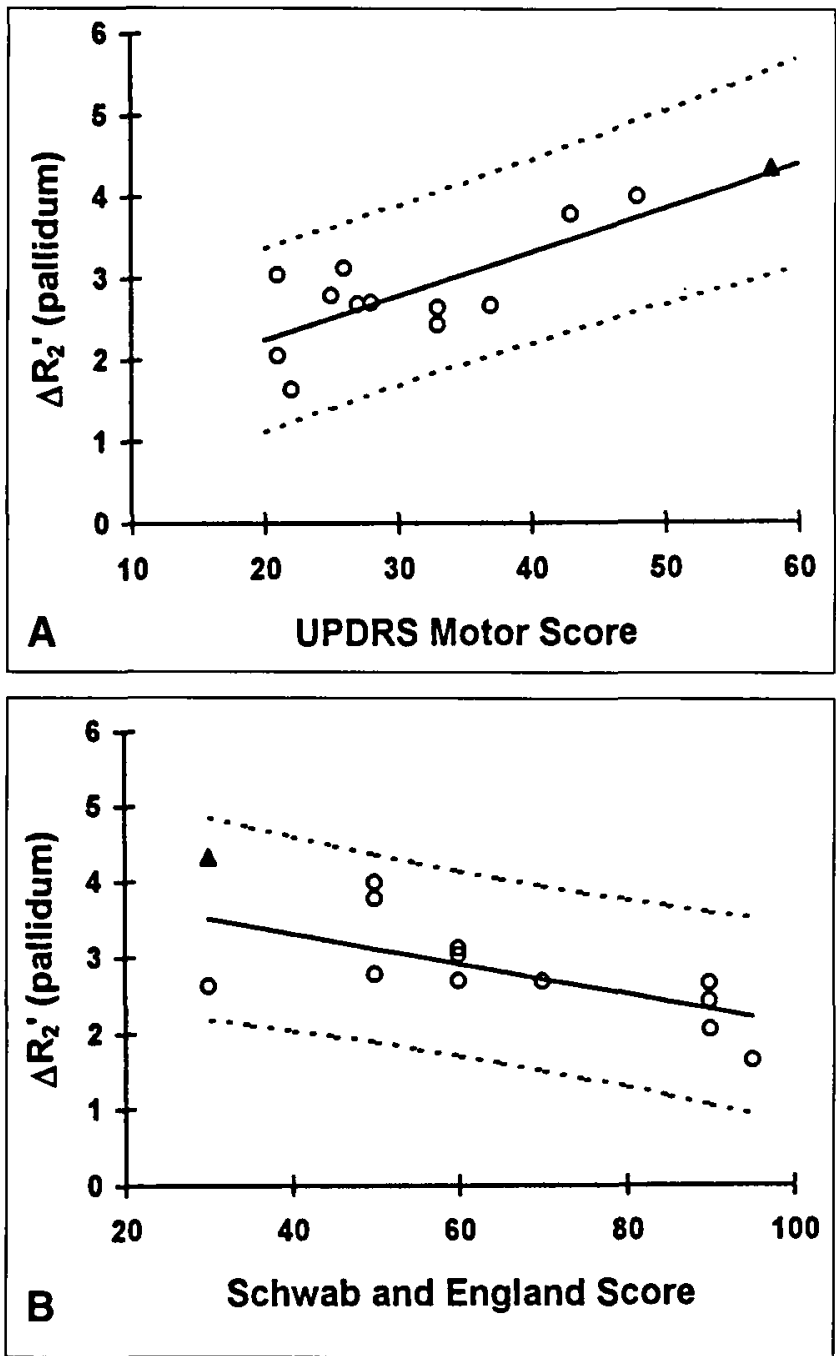

Figure 2: $\Delta R_{2}^{\prime}$ (iron content) in pallidum in Parkinson's disease (open circles) and a patient with probable striatonigral degeneration (closed triangle) as a function of UPDRS motor score (a) and Schwab and England activities of daily living score $(b)$. The regression line and 95\% confidence limits for inclusion in the Parkinson's disease group are indicated. Parkinson's patients are from reference 6.

with clinical disease severity in PD $^{6}$ and because our patient had advanced clinical features, we compared these measurements to the expected range of $\Delta R_{2}{ }^{\prime}$ values in PD occurring as a function of disease severity (see Figures $I$ and 2). Even when considering clinical severity as indicated by the motor subscale of the UPDRS $^{12}$ and by the Schwab and England activities of daily living score, ${ }^{13}$ the putamenal $\Delta R_{2}{ }^{\prime}$ value in our patient exceeded the 95\% confidence limit for inclusion in the PD group (see Figure 1). In contrast, the pallidal value remained well within the $95 \%$ confidence limit for inclusion in the PD group (see Figure 2).

\section{Discussion}

Our patient presented with a sporadic, adult-onset parkinsonian syndrome consisting primarily of bradykinesia and rigidity, which was initially responsive to levodopa. With disease progression, however, the parkinsonian features worsened substantially, became very poorly responsive to levodopa, and became associated with evidence of symptomatic autonomic failure.
These features are consistent with a diagnosis of probable multiple system atrophy, SND type. ${ }^{14}$

A correlation between neuropathological and MRI abnormalities in SND has been reported previously. ${ }^{15-17}$ These reports indicate a "slit-like void signal" in the putamen on $T_{2}$ weighted images in patients with the typical neuropathological findings of SND. Lang et al. reported a correlation between this signal loss and increased iron deposition in the putamen demonstrated post mortem by Perls' stain. ${ }^{16}$ In contrast, Schwarz et al. observed an imperfect correlation between putamen iron deposition and low signal changes on $T_{2}$ images with increased iron in only one of two patients reported. ${ }^{17}$ Some, but not all, patients also appear to have a thin band of high signal change in the lateral putamen. ${ }^{17}$ Our results are consistent with these previous reports and indicate that in vivo estimates of putamenal iron content with MR may be useful in confirming a diagnosis of SND in the appropriate clinical setting.

These observations suggest that putamenal pathology is particularly prominent in this disorder. This is consistent with the established neuropathological findings of neuronal loss and reactive gliosis in the putamen in SND, and with the previous report of Lang et al. ${ }^{16}$ of a high concentration of iron occurring within the putamen. This is also consistent with the observations of Eidelberg et al. ${ }^{18}$ who have demonstrated substantial abnormalities in the putamen with positron emission tomography, with severe putamenal hypometabolism in SND as opposed to the normal putamenal metabolism which is typical of PD. Although neuronal loss and gliosis may also be present in the pallidum, our observation that changes in iron content are similar in severity to that seen in idiopathic PD suggests that pathological and/or radiological changes in this structure may be less specific for SND in contrast to changes in the putamen. Significant neuronal loss with accompanying gliosis is also typically present in the substantia nigra in SND. We were unable to address the possibility of altered nigral iron content because of the small size of this structure and the sensitivity of our MR method to artifacts resulting from small head movements. Others, however, have reported increased iron levels in both the substantia nigra and the putamen in SND. ${ }^{19}$

Striatonigral degeneration is primarily a neuropathological diagnosis, although there is a typical complex of clinical features which may be very suggestive of this disorder. Our results suggest that the in vivo estimation of regional brain iron content with appropriate MR methods may be a useful adjunct in the investigation of patients with atypical PD. The specificity and sensitivity of these changes which we have observed, however, require further study.

\section{ACKNOWLEDGEMENTS}

This project was supported in part by the Parkinson Foundation of Canada and the H.M. Toupin Medical Foundation. The assistance of Joanne Hodder, B.S.N., is appreciated.

\section{REFERENCES}

1. Hughes AJ, Daniel SE, Kilford L, Lees AJ. Accuracy of clinical diagnosis of idiopathic Parkinson's disease. J Neurol Neurosurg Psychiatry 1992; 55: 181-184.

2. Stern MB, Braffman BH, Skolnick BE, Hurtig HI, Grossman RI. Magnetic resonance imaging in Parkinson's disease and parkinsonian syndromes. Neurology 1989; 39: 1524-1526. 
3. Pasatakia B, Polinsky R, De Chiro G, et al. Multiple system atrophy (Shy-Drager syndrome): MR imaging. Radiology 1990; 174: 609-696.

4. Drayer BP, Olanow W, Burger P, et al. Parkinson plus syndrome: diagnosis using high field MR imaging of brain iron. Radiology 1986; 159: 493-498.

5. Gorell JM, Ordidge RJ, Brown GG, et al. Increased iron-related MRI contrast in the substantia nigra in Parkinson's disease. Neurology 1995; 45: 1138-1143.

6. Ye FQ, Allen PS, Martin WRW. Basal ganglia iron content in Parkinson's disease measured with magnetic resonance. Mov Dis 1996; 11:243-249.

7. Folstein MF, Folstein SE, McHugh PR. "Mini-mental state": a practical method for grading the mental state of patients for the clinician. J Psychiatr Res 1975; 12: 189-198.

8. Ye FQ, Allen PS. Ferritin as a susceptibility agent. In: Proceedings of the 12th Annual Meeting of Society of Magnetic Resonance in Medicine. Berkeley: Society of Magnetic Resonance, 1993: 800.

9. Ye FQ, Allen PS. Relaxation enhancement of the transverse magnetization of water protons in paramagnetic suspensions of red blood cells. Magn Reson Med 1995; 34: 713-720.

10. Ye FQ, Martin WRW, Allen PS. Estimation of brain iron in vivo by means of the interecho time dependence of image contrast. Magn Reson Med 1996; 36: 153-158.

11. Ye FQ, Martin WRW, Allen PS. Estimation of the iron concentration in excised grey matter by means of proton relaxation measurements. Magn Reson Med 1996; 35: 285-289.
12. Fahn S, Elton RL. Unified Parkinson's disease rating scale. In: Fahn $S$ et al., eds. Recent Developments in Parkinson's Disease, Vol II. Florham Park: Macmillan, 1987: 153-163.

13. Schwab RS, England AC. Projection technique for evaluating surgery in Parkinson's disease. In: Gillingham FJ, Donaldson IML, eds. Third Symposium on Parkinson's Disease. Edinburgh: Livingstone, 1969: 152-157.

14. Wenning GK, Ben Shlomo Y, Magalhaes M, Daniel SE, Quinn NP. Clinical features and natural history of multiple system atrophy. An analysis of 100 cases. Brain 1994; 117: 835-845.

15. O'Brien C, Sung JH, McGeachie RE, Lee MC. Striatonigral degeneration : clinical, MRI and pathological correlation. Neurology 1990; 40: 710-711.

16. Lang AE, Curran T, Provias J, Bergeron C. Striatonigral degeneration : iron deposition in putamen correlates with the slit-like void signal of magnetic resonance imaging. Can J Neurol Sci 1994; 21: $311-318$

17. Schwarz J, Weis S, Kraft E, et al. Signal changes on MRI and increases in reactive microgliosis, astrogliosis, and iron in the putamen of two patients with multiple system atrophy. J Neurol Neurosurg Psychiatry 1996; 60: 98-101.

18. Eidelberg D, Takikawa S, Moeller JR, et al. Striatal hypometabolism distinguishes striatonigral degeneration from Parkinson's disease. Ann Neurol 1993; 33: 518-527.

19. Kato S, Meshitsuka S, Ohama E, et al. Increased iron content in the putamen of patients with striatonigral degeneration. Acta Neuropathologica 1992; 84: 328-330. 